\title{
Microsatellite attitude control approach: combined with Generation Adversarial Networks fault-detection and Cerebellar Model Articulation Controller fault-tolerant control
}

\author{
Ho-Nien Shou \\ Dep. of Aviation \& Communication Electronics, Air Force Institute of Technology \\ Gangshan, Kaohsiung, Taiwan (R.O.C.)
}

E-mail: honien.shou@gmail.com

\begin{abstract}
In this paper, a new attitude control architecture for microsatellite is proposed. Based on the deep learning faultdetection method, the Cerebellar Model Articulation Controller (CMAC) is used as the fault-tolerant control. With the function of Generation Adversarial Networks (GAN) to recognize images, the microsatellite attitude fault wavelet spectrum is used as a guide for the training of generators and discriminators, and for system real-time fault diagnosis and classification. When the system fault diagnosis determines that the fault occurs, the cerebellar neural network is involved in fault-tolerant control. The GAN learning ability of the generative confrontation network is used to solve the problem of insufficient sample data and sample labeling respectively. The CMAC is used as a local learning network, which has generalization ability, strong convergence speed, and easy software and hardware implementation. The simulation results show that compared with other methods, the GAN method of fault-detection combined with CMAC can achieve higher accuracy and robustness.
\end{abstract}

Keywords: deep learning, fault-detection, fault-tolerant control, Cerebellar Model Articulation Controller, Generation Adversarial Networks.

\section{Introduction}

The Generative Adversarial Network (GAN) was proposed in 2014 [1]. The GAN continuously optimizes the network to achieve better results by using the mutual confrontation between generator and discriminator. There are many hidden deep learning network models in GAN, and it has good feature learning ability. At first, it is mainly used in data generation and image generation to solve the problem of insufficient sample data. Based on different research purposes, derivative models for GANs are constantly being proposed. A SemiSupervised Learning with Context-Conditional Generative Adversarial Networks (SSL) is proposed, in which the generator structure remains unchanged and the discriminator is classified in two categories an output layer is added to achieve the multi classification function [2]. Since the SSL was proposed, it has been applied to speech generation and recognition, image recognition, sample classification and other fields [3]. The SSL is applied to human action recognition, and the images are captured by the video frame as the network input for action recognition [4]. In the above application, the SSL achieves the recognition effect.

The Cerebellar Model Articulation Controller (CMAC) was developed by Dr. J.S. Albus in 1975, based on the biological model of cerebellar cortex proposed by Marr in Medical Research Institute. CMAC are widely used because of characters of fast convergence speed, strong generation ability, it can overcome the local optimization problem of back propagation neural network (BP), simple structure, easy implementation by

(C) The 2021 International Conference on Artificial Life and Robotics (ICAROB2021), January 21 to 24, 2021 
software and hardware. CMAC has been successfully applied in many application fields, such as fault detection and nonlinear control. In the classification task, Lin et al. [5], a parameter fuzzy CMAC neural network with mixed parameters is proposed. Learning algorithm composed of self-clustering genetic algorithm (GA) and improved GA for facial detection and breast cancer diagnosis.

In recent years, the research on motor bearing fault diagnosis has been widely concerned by scholars and engineers. The vibration analysis method is commonly used for bearing fault diagnosis, which analyzes and classifies the collected bearing vibration signals. However, bearing vibration signal has the characteristics of non-stationary, so it is very difficult to extract the characteristics of the bearing.

As a time-frequency analysis method, continuous wavelet transform can effectively extract the timefrequency characteristics of non-stationary signals. The function of wavelet transform is to take one-dimensional signal as input. The result of time-frequency analysis essentially reflects the two-dimensional spectrum of energy intensity of signal at different time and frequency, and the output is displayed in the form of time-frequency diagrams. At present, continuous wavelet transform has been widely used in the field of mechanical fault diagnosis [6]. In reference, a combination of wavelet transform branch support vector machine (SVM) and convolutional neural networks $(\mathrm{CNN})$ was proposed. In reference [6], wavelet transform and back propagation neural network (BP) were used to realize fault diagnosis of different motors. In the application of the above literature, the feature vector is constructed artificially based on the time-frequency map, and the learning and expression ability of the selected neural network is limited [6].

The content structure of this paper is a brief introduction to Section 1 and a description of SSL in Section 2 in Section 3, the CMAC is explained, in Section 4 wavelet transform of fault model, in Section 5 attitude fault diagnosis and fault-tolerant control simulation of microsatellite in Section 6. Finally, the conclusion is drawn.

\section{Semi-Supervised Learning with Context- Conditional Generative Adversarial Networks (SSL)}

The SSL routing generator and discriminator are composed of the generator and discriminator. The generator belongs to the generative model. The difference between the generator and other models is that the generator does not need to be modeled in advance. However, due to the lack of constraints, the generated data is too free. This paper proposes a kind of improvement to solve the above problems Based on the semi supervised generative countermeasure network algorithm, conditional variable is added to the model to guide the training process of generator and discriminator by using additional information. Compared with the prototype generated countermeasure network, the main difference is that the discriminator outputs a class signal (the generated sample is No For the discriminator, the loss function consists of two parts, one is supervised learning loss (only need to judge whether the sample is true or false), and the other is unsupervised learning loss (judging sample category). The generator only needs to generate realistic samples as far as possible. After training, the discriminator can be used as a classification model to classify.

The generator of the improved SSL consists of several deconvolution layers, excitation layers and extracted over fitting Fitting) and speeding up the training speed method, for the input $n$-dimensional noise signal connecting condition variables, the final output is almost the same as the real signal "false" signal, which can achieve the state of "false" with the real signal, which is conducive to expand the sample to achieve better training effect. The discriminator consists of several convolution layers, excitation layers, fully connected layers and classified output layers, as well as the

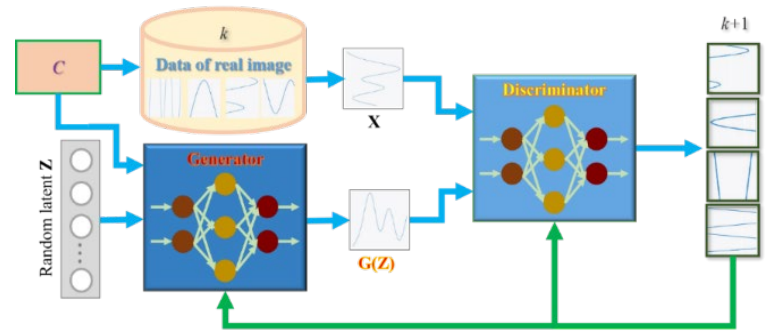

Fig. 1. SSL algorithm process and structure corresponding methods to prevent over fitting and speed up the training. The discriminator takes the real signal, that is, the signal to be detected and the "false" signal generated by the generator, as the input at the same time. 
The flow chart of the improved generative countermeasure network algorithm is shown in Fig. 1.

\section{Cerebellar Model Articulation Controller (CMAC)}

CMAC is a kind of artificial learning network which imitates the hierarchical storage of information in human cerebellar cortex, and its operation process is basically achieved by a series of mapping methods. As shown in Figure 2, the basic structure of MIMO CMAC with three inputs and three outputs is shown Fig. 2.

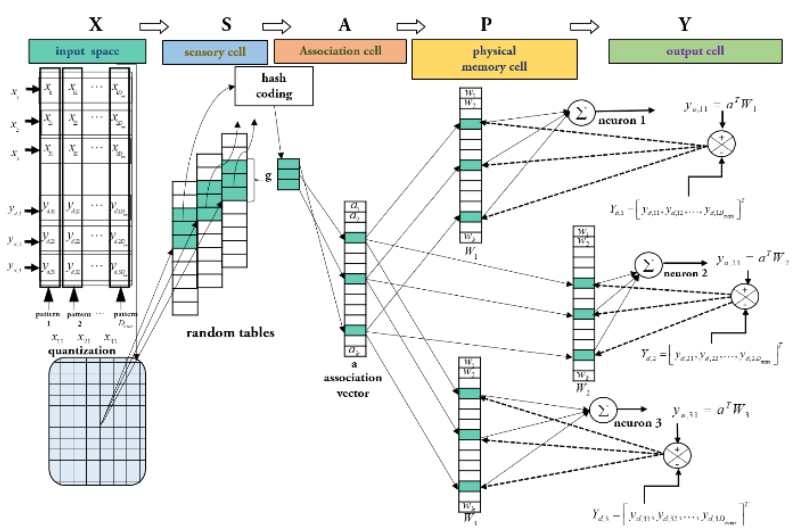

Fig. 2. The MIMO CMAC block diagram

The five units of CMAC neural network consist of (i) input space (X), (ii) sensory cell (s), (iii) Association unit (A), (iv) physical storage unit (P) and (v) output storage cell (Y) using a series of images. Fig. 2 shows the network topology vector of three inputs and three output vectors of CMAC neural network; the input is represented by $\left[x_{1}, x_{2}, x_{3}\right]^{T}$, and the actual output is $\left[y_{d 1}, y_{d 2}, y_{d 3}\right]^{T}$. The figure shows an icon for the input mode 1 image, including three input elements $\left[x_{1}, x_{2}, x_{3}\right]^{T}$ and one desired output $y_{d 1}$.

\section{Fault Model Wavelet Transform}

The attitude fault diagnosis method of microsatellite is based on the fault signal generated by deep convolution and combined with continuous wavelet transform. The ability of wavelet transform for nonstationary signal and the function of SSL processing and image recognition are introduced. Based on the SSL, the model is introduced and the loss function is optimized and optimized. In order to guide the training of generator and discriminator, the generator and semi supervised learning ability are used to solve the problem of sample data shortage and sample marking respectively.

Case1.

$$
\begin{aligned}
& f_{a_{1}}=\left\{\begin{array}{l}
0.5,40 \leq t<70(\mathrm{sec}) \\
0
\end{array}\right. \\
& f_{a_{2}}=f_{a_{3}}=0
\end{aligned}
$$

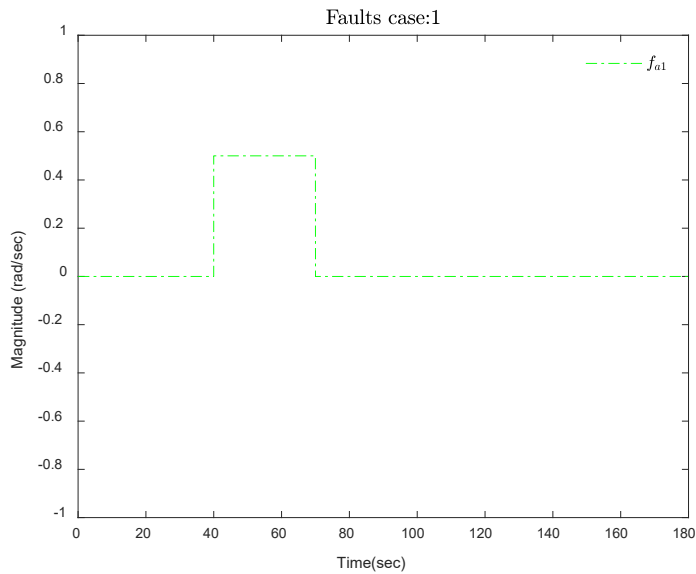

Fig. 3 Fault case 1

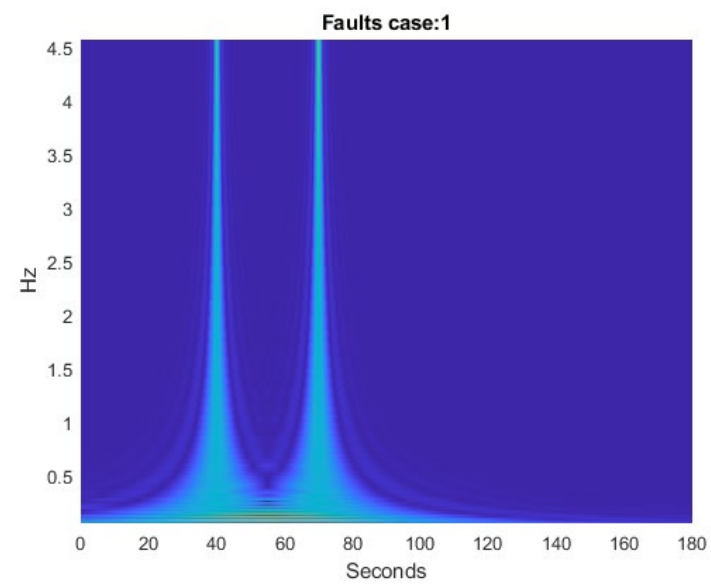

Fig. 4 wavelet transform coefficient diagram of case 1 attitude fault

The fault signal is transformed into frequency time wavelet coefficient graph (time-frequency diagram) to achieve time-frequency analysis of vibration signal and obtain more comprehensive and detailed information. 
The feature extraction and image recognition ability of deep learning GAN are used, and the compressed timefrequency diagram is used as input. The wavelet transform coefficients of case 1is shown in Fig. 4.

\section{Fault Estimated and Fault Tolerant Control Simulation of Microsatellite Attitude}

The microsatellite dynamic equation is expressed as a nonlinear equation

$$
J \omega=-\omega^{\times} J \omega+\tau_{w}+\tau_{u}
$$

where $J=\operatorname{diag}\left\{J_{x}, J_{y}, J_{z}\right\}$ are inertia moments of the satellite along principal axes; $\boldsymbol{\omega}$ are the angular velocity of the body-fixed reference frame. $\boldsymbol{\tau}_{w}$ are space disturbance torques and $\boldsymbol{\tau}_{u}$ are the control torques along principal axes.

For small attitude angles, the derivation of eq. (2) is as follows

$$
\left\{\begin{array}{l}
\dot{x}(t)=A x(t)+g(t, x)+B_{u} u(t)+B_{w} w(t)+F_{a} f_{a}(t) \\
y(t)=C x(t)+F_{s} f_{s}
\end{array}\right.
$$

Where state vector $x=\left[\begin{array}{llllll}\psi & \theta & \phi & \dot{\psi} & \dot{\theta} & \dot{\phi}\end{array}\right]^{T}$, nonlinear term $g(t, x)=\left[\begin{array}{c}0 \\ \omega_{0} \\ 0 \\ \left(J_{z}-J_{y}\right) \omega_{y} \omega_{z} / J_{x} \\ \left(J_{x}-J_{z}\right) \omega_{x} \omega_{z} / J_{y} \\ \left(J_{y}-J_{x}\right) \omega_{x} \omega_{y} / J_{x}\end{array}\right]$, actuator fault matrix $F_{a}=\left[\begin{array}{cccccc}0 & 0 & 0 & 1 & 0 & 0 \\ 0 & 0 & 0 & 0 & 1 & 0 \\ 0 & 0 & 0 & 0 & 0 & 1\end{array}\right]^{T}$ and actuator fault vector $f_{a}=\left[\begin{array}{lll}f_{a_{1}} & f_{a_{2}} & f_{a_{3}}\end{array}\right]^{T}$.

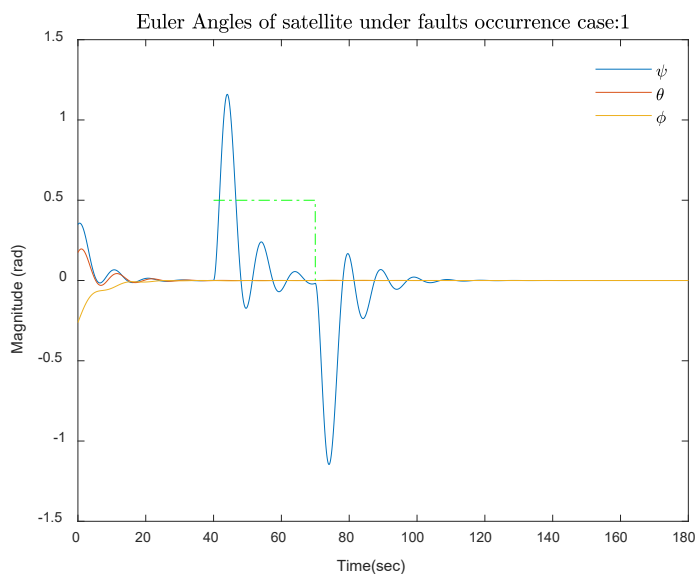

Fig. 5 Time response of case 1 attitude angle failure and recovery

\section{Conclusion}

According to the numerical simulation results in the previous section, the feasibility of applying SSL to microsatellite fault estimated. Through SSL of GAN samples and training parameters, the CMAC has good generalization ability and stability under the brake fault model.

\section{References}

1. Ian J. Goodfellow, Jean Pouget-Abadie, Mehdi Mirza, Bing $\mathrm{Xu}$, David Warde-Farley, Sherjil Ozair, Aaron Courville and Yoshua Bengio, "Generative adversarial nets," In Advances in Neural Information Processing Systems(NIPS), 2014, pp.2672-2680.

2. Salimans T., Goodfellow I., and Zaremba W., "Improved techniques for training GANs," In NIPS, 2016, pp. 2234 2242.

3. Salimans T, Goodfellow I, Zaremba W, et al., " Improved techniques for training GANs," Advances in Neural Information Processing Systems, 2016, pp. 2234-2242.

4. Lantao $\mathrm{Yu}$, Weinan Zhang, Jun Wang, Yong Yu, "SeqGAN: Sequence Generative Adversarial Nets with Policy Gradient," 31th AAAI Conference on Artificial Intelligence, , 2017, pp.2852-2858.

5. C.J. Lin, J.H. Lee, C.Y. Lee, A novel hybrid learning algorithm for parametric fuzzy CMAC networks and its classification applications, Expert Systems with Applications 35, 2008, pp.1711-1720.

6. Yuan Jian-hu, Han Tao, Tang Jian, et al. "An approach to intelligent fault diagnosis of rolling bearing using wavelet time-frequency representations and CNN," Machine Design and Research 33(2), 2017, pp.93-97. 